\title{
Hemophagocytic Lymphohistiocytosis Syndrome in Dengue Hemorrhagic Fever: Authors' Reply
}

\author{
S. Raju • Shantaraman Kalyanaraman • \\ K. Swaminathan $\cdot$ A. Nisha $\cdot$ S. Praisid
}

Received: 2 August 2014 / Accepted: 3 December 2014 / Published online: 16 January 2015

(C) Dr. K C Chaudhuri Foundation 2015

To the Editor: This is in reply to the letter by B. Joob and V. Wiwanitkit on our study entitled "Hemophagocytic lymphohistiocytosis syndrome in dengue hemorrhagic fever" [1]. HLH is a life-threatening complication with variable clinical presentation [2] and is secondary to infections (viral, bacterial, fungal or protozoan) in tropical countries. In an Indian report by Ramachandran et al., dengue was reported as the leading cause for HLH [3]. Veerakul et al. also reported a similar experience [4]. Persistence of fever beyond 7-8 $d$ in dengue is usually due to secondary sepsis or dengue associated HLH. In our study, all 23 children presented with fever of more than $8 \mathrm{~d}$, malena in $86.95 \%$, organomegaly in all children, progressive cytopenias and abnormal chemistry, namely triglyceride, fibrinogen and ferritin. All children admitted to the intensive units had blood and urine cultures done as a routine and only those children who did not have bacterial growth in both were taken for the study. That excludes children with sepsis. In areas where dengue is endemic, the awareness of dengue-associated HLH as a cause of fatality, needs to be highlighted as its course is similar to severe dengue and sepsis induced multi organ dysfunction, but responds well to steroids and IV immunoglobulin.

Conflict of Interest None.

Source of Funding None.

\section{References}

1. Raju S, Kalyanaraman S, Swaminathan K, Nisha A, Praisid S. Hemophagocytic lymphohistiocytosis syndrome in dengue hemorrhagic fever. Indian J Pediatr. 2014. doi:10.1007/s12098-014-1425-4.

2. Pal P, Giri PP, Ramanan AV. Dengue associated hemophagocytic lymphohistiocytosis: a case series. Indian Pediatr. 2014;51:496-7.

3. Ramachandran B, Balasubramanian S, Abhishek N, Ravikumar KG, Ramanan AV. Profile of hemophagocytic lymphohistiocytosis in children in a tertiary care hospital in India. Indian Pediatr. 2011;48:31-5.

4. Veerakul G, Sanpakit K, Tanphaichitr VS, Mahasandana C, Jirarattanasopa N. Secondary hemophagocytic lympho-histiocytosis in children: an analysis of etiology and outcome. J Med Assoc Thail. 2002;85:S530-41.

\footnotetext{
S. Raju $\cdot$ A. Nisha $\cdot$ S. Praisid

Department of Pediatrics, Krishna Hospitals, Tirunelveli, Tamilnadu, India

S. Kalyanaraman $(\bowtie) \cdot K$. Swaminathan

Department of Pathology, Tirunelveli Medical College, Tirunelveli,

Tamilnadu 627001, India

e-mail: ananthsaketh@gmail.com
} 